\title{
Emopad: An Affective Gamepad
}

\author{
Pedro H. F. Bacchini \\ Department of Computing \\ Pontifical Catholic University of \\ Goiás, PUC-Goiás \\ Goiânia, Brazil
}

\author{
José Olímpio Ferreira \\ Department of Computing \\ Pontifical Catholic University of \\ Goiás, PUC-Goiás \\ Goiânia, Brazil
}

\author{
Talles Marcelo G de A. \\ Barbosa \\ Department of Computing \\ Pontifical Catholic University of \\ Goiás, PUC-Goiás \\ Goiânia, Brazil
}

\begin{abstract}
Affective Systems have been used in different applications, such as stress monitoring in aircraft seats and managing sensitivity in autism spectrum disorder. Although many scientific progresses have been made there are many computational challenges to be overcome in order to embedded affectivity into traditional user interfaces. For example, context-sensitive algorithms, low-complexity pattern recognition models and hardware customizations are requirements to support the simplification of user's experience becoming more intuitive, transparent and less obstructive. In this paper an affective gamepad is presented. This acquisition system has been developed to improve user's biofeedback when they are playing games on Microsoft Xbox or Sony PlayStation. The preliminary version of Emopad is able to capture Galvanic Skin Response (GSR), Temperature, Force, Heart Rate (HR) and its variability (HRV) by photoplethysmography (PPG) sensor while complementary algorithms are executed to filter noise, recognize interests patterns and classify events related to user's emotional states. All processing phases are embedded into Emopad, and they explore microcontroller's dynamic power management. Emopad has been developed to deal with energy-efficient and platform-independent requirements. Concerning to this paper's aim, the acquisition systems development is highlighted. Also, the sliding window-based algorithm is presented and evaluated. It has been applied after sampling in GSR, Force, Temperature and HR signals to detect events related to emotional responses. The success of affective gamepads can lead to a paradigm shift, because traditional consoles can be configured to work as Point-of-Care technologies. Consequently, they can receive, process and transmit physiological data and events related to clinical conditions about their users.
\end{abstract}

\section{General Terms}

Embedded Systems, Affective Systems, Biofeedback Systems

\section{Keywords}

Affective Gamepad; Affective Systems;

\section{INTRODUCTION}

The development of computer systems including new user's requirements, such as emotional states has been largely discussed for the last two decades [1] [2] [3] [4], since Rosalind Picard coined the term Affective Computing at MIT Media Labs in 90's. The ability of machines to recognize emotions is an important step to improve the HumanComputer Interactions (HCI). Thus, decision-making could become more intuitive and simplified. Many effort has been applied to recognize emotions through facial expressions, voice and physiological signals in different applications, e.g., embedded in aircraft seats [5], smartphones [6] [7], wearable computers [8] [9], cars [10], [11], [12], robots [13] and even embedded in a bathroom's mirror [14]. Recently, more interest in clinical, educational and entertainment applications have been observed [15] [16] [17].

The game industry has special interest in affective games because all video games have the ability to affect a player and the result can be seen in changes of his or her focus or concentration, arousal and stress levels. Virtual stimuli can produce angry, anguish, sadness, panic, fear, happiness, joy, well-being and etc. Most of these emotional states can be expressed directly or indirectly on physiological signals, e.g., Heart Rate (HR) and its variability (HRV), Galvanic Skin Response (GSR), Respiration, Cutaneous Temperature, Movement, Force, ECG, EEG and etc. In the last decade, there has been increased commercial interest in using physiological input in digital games, not only for biofeedback training, but also to enhance immersion and engagement for players [18].

Schaaf and Adam [19] have used an HRV sensor as indicator of emotional arousal for mobile games. For some features, it is possible to use ultra-short term window sizes (shorter than 5 minutes) for short term HRV analysis. Parnandi et al. [20] have experimented electrodermal activity (EDA) as a physiological correlate of arousal on car-racing games. Kennedy et al. [21] have developed a low-cost rehabilitation instrument suite centered around the Nintendo Wii Balance Board that has the potential to enhance rehabilitation for patients with balance disorders. Balance retraining is a critical part of rehabilitation for many individuals following neurotrauma such as stroke. Nacke et al. [22] propose taxonomy based on direct and indirect physiological sensor input to expand traditional game control. Direct physiological control establishes that any user can manipulate and control directly (e.g., muscle flexion, eye gaze) and should be mapped intuitively to reflect an action in the virtual world. Indirect physiological inputs are excellent at helping users train themselves to relax precisely because they are indirectly controlled, such as GSR and HRV signals. In May 2014 was launched a game entitled "Throw Trucks". According to [23] it allows users that use NeuroSky's MindWave to give them telekinetic super-powers controlled with their thoughts. NeuroSky's MindWave is one-channel acquisition headset able to capture and process electroencephalogram (EEG). In Throw Trucks, users can crush their enemies by throwing trucks with their minds. Recently, a posted headline at IEEE Spectrum website announces the development of a modified Xbox controller for sensing players' excitement levels at Stanford University [24]. However, they have not submitted any paper for peer review yet. 
In this paper a biofeedback device is presented. Also, a complete description about its development process, based on bottom-up approach, and starting at acquisition systems development activity in order to generate data sets and usability parameters to guide subsequent activities. It has been developed to operate as a user's affective interface, able to deal with implicit and explicit biofeedback processing. Consequently, it has been called Emotional Gamepad or Emopad. Furthermore, energy-efficient and platformindependent requirements were required. Hence, a generic game controller has been redesigned to embed sensors, processor and algorithms able to capture, filter, recognize and classify events, especially those related to user's emotional states when they are playing video games. We argue that embedding these new functionalities in generic game controllers became them more intuitive (preserving traditional functionalities) and useful (platform-independent). Moreover, the proposed game controller device should transform a generic gamepad into a point-of-care technology bringing a personal health monitor system at or near the site of patient care.

\section{MATERIALS AND METHODS}

\subsection{Development Process}

In order to develop Emopad a bottom-up and successive refinements development methodology was applied. This sort of process is recommended when most of requirements cannot be completely elicited. These requirements are partially observed. Thus, basic and low-level components should be built. As soon as possible they should be customized and fused to arrange high complex and more precise components. Fig. 1 synthetizes applied methodology.

The Acquisition Systems were the first component implemented. Their developments were essentials to evaluate, select and handle with physiological signal processing requirements, such as patterns of interests, dimensions, costs, computational complexity and ergonomics aspects. In a preliminary version of Emopad, Galvanic Skin Response (GSR), Cutaneous Temperature, Force, Heart Rate (HR) and its variability (HRV) have been captured and analyzed. In addition, outside of the proposed gamepad, HD Webcam, voice (from each player participant), ambient temperature, ambient humidity and game sequence (in video format) should be also recorded. Fig. 5 shows external variables. Moreover, many DSP technologies were tested and evaluated to support non-functional requirements, such as low cost, platform-independence, ergonomics aspects (short dimensions) and energy efficiency, very important for batteryoperated devices.

After the first activity (Fig. 1) two parallels activities were initiated. The development of Analysis, Visualization and Dataset Tools activity was responsible for organizing acquired data preparing it for analyzing and visualizing. Both functionalities are indispensable to design algorithms qualified to perform filter noise, recognize interests patterns and classify events. In this activity, all parameters related to embedded algorithms were defined, e. g., window size, resampling rate, outlier's references and decision rules. The Redesign Gamepad activity was executed to establish ergonomic parameters to accommodate physical interfaces and their related circuitry. Hence, 3-D modeling was executed based on traditional gamepad models. Before to produce 3-D software models and their artifacts, some 3-D models sculpted with epoxy putty were created. They were built to be touched and assessed by selected user's set at preliminary usability testing. Consequently, the new gamepad surface, colors, curves, sensors and actuators could be tangibly evaluated. In this activity many hypothesis were tested and final version was inspired in a bike handlebar from italian bianchi, circa 1940. At the end of Redesign Gamepad activity a 3-D plastic model was printed at Shapeways spinoff in New York City [25].

After to design and evaluate certain algorithms and heuristics, the best performance solutions have been selected and embedded in a microcontroller platform. The best performance choice was based on a compromise between algorithms hit rate, time/space complexity and energy efficiency. Thus, at the end of Algorithm Implementation activity at least one feasible hardware-software solution was achieved.

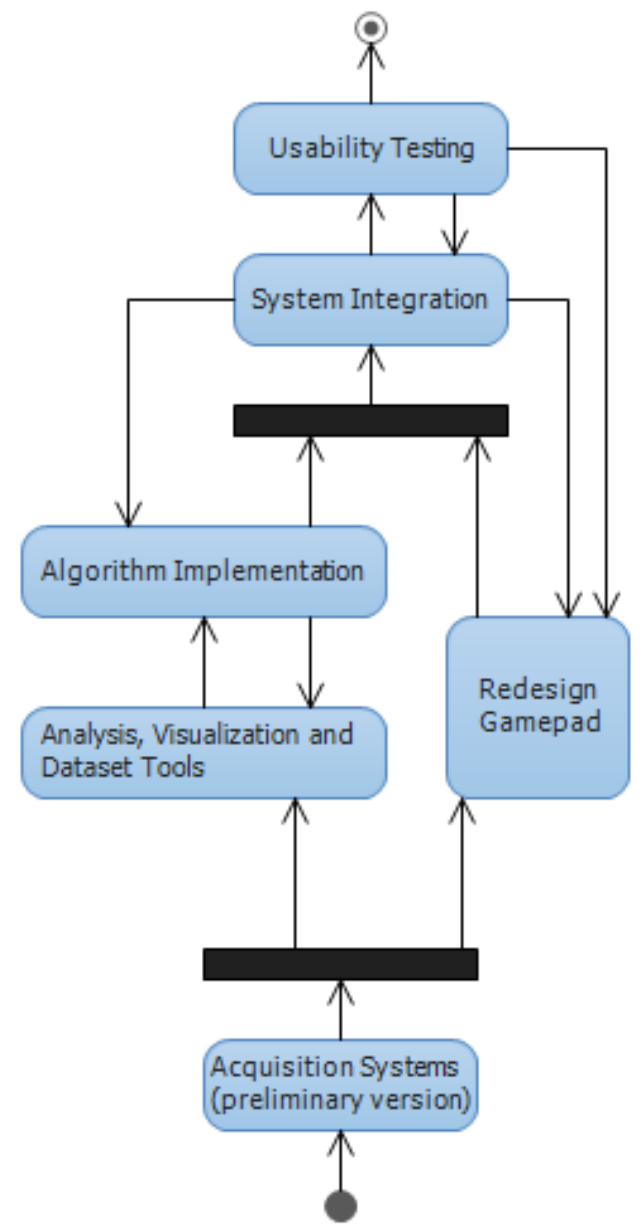

Fig 1: Emopad Development Process

The last two activities in Fig. 1 are related to production and evaluation of an operational prototype version. They should embrace hardware-software artifacts embedded in the Gamepad enclosure and usability testing to verify user's reactions. Most of performed activities required feedback for refactoring non-conforming modules, e.g, programs, electronic circuits, 3-D models, algorithms simulations and analysis. These hypotheses were considered in the proposed development process by each arrow directions in Fig. 1.

\subsection{Acquisition Systems}

The Emopad acquisition's systems were first prepared to deal with four analog signals: GSR, Cutaneous Temperature, Force and PPG. Four different analog interfaces were built and connected to Analog/Digital (A/D) converter embedded in 
MSP430 TI microcontroller. Fig. 2 shows the block diagram to clarify Emopad hardware organization and Fig. 5 shows the first prototype version build according to Fig. 2 .

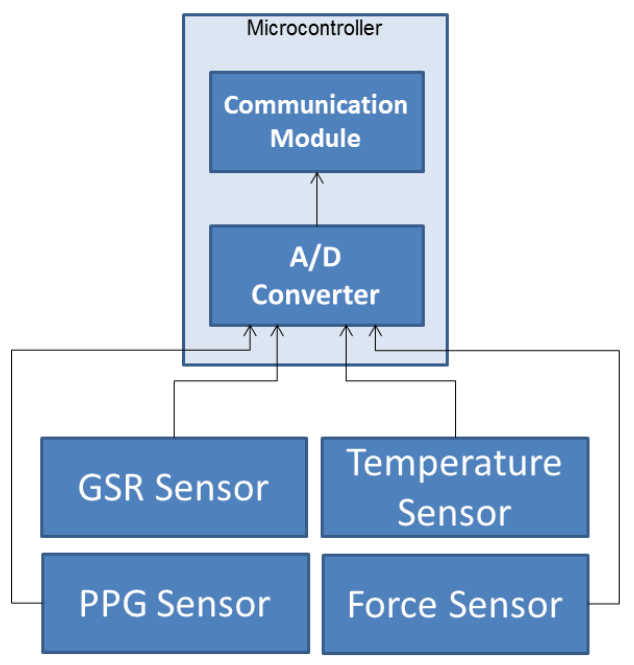

\section{Fig 2: Block Diagram}

According to [26] [27] GSR reflects psychological stimuli and it has linear correlation with arousal, exposing some user's emotional states. Fig. 3 shows the GSR circuitry developed to embedded it into Emopad. It has been described in previous papers [4], and it works as a voltage divisor, useful to compare a known resistance value with the skin resistance at each time. In the calibration phase, the " $2.7 \mathrm{M} \Omega$ " resistance should be fine-tuned according to the maximum skin resistance for each person at each moment. Buffers are implemented by two ampops and two analog filters, and they were applied to remove undesired artifacts (specially those related to high frequency noise), to avoid the lost of signal strength and to limit the bandwidth, preventing aliasing. In this paper the GSR circuitry has been designed to be embedded in a gamepad, enabling it to capture GSR data using only two fingers on left hand. Although a recent Picard's paper [28] suggests the need to capture GSR in many and different body parts, we argue that a Sensor Network based solution is not feasible to Emopad because that approach could become obstructive and CPU bound. Most entertainment applications related to affective interfaces are concerning in capture the event occurrences (at low level) and fuse them with the other events from different sensors. Next, data should be prepared for pattern recognizing and decisionmaking (at high level algorithms), commonly executed outside of the microprocessor embedded into affective interfaces. This situation prevents platform independence because these functionalities should be implemented at driver level on game consoles. Also, data redundancy provided by sensor networks or long term monitoring systems is more critical to clinical applications than entertainment application of affective computing.

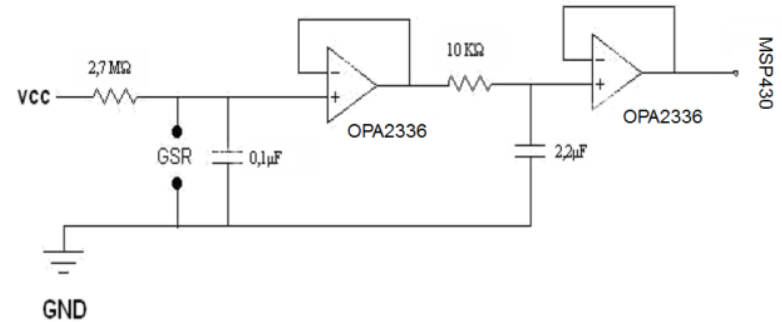

Fig 3: GSR Circuitry

Fig 4 shows the cutaneous temperature circuitry. It has been designed to avoid first-degree burns when the current through the thermistor increases. In order to reach this objective the REF200 from Texas Instruments was chose. It implements a high precision dual current source. This approach prevents the self-heating error from thermistor [29]. Other functional blocks used in Fig. 4 are one differential amplifier, one buffer, one voltage reference generator, two filters and one thermistor. This circuit was adjusted to operate at $5 \mathrm{~V}$ and the total sink current is less than $4 \mathrm{~mA}$. Thus, a voltage divider must be used at circuit output. Temperature range was regulated from $10^{\circ} \mathrm{C}$ to $40^{\circ} \mathrm{C}$. Also the B57861S0103F045 was used; arrows on Fig. 5 point it. This thermistor is manufactured by Epcos [30] and has a short response time, $1 \%$ resistance tolerance. It is very small and therefore easy to fit in the gamepad. Its leads are insulated what prevent current to flow to the body. Different from the circuitry proposed in [29], this circuitry uses just one current source per channel. Thus, just one REF200 is necessary for each two channels, reducing costs when more than one channel is required for cutaneous temperature. In the first version of Emopad, two channels were used to capture data related to cutaneous temperature.

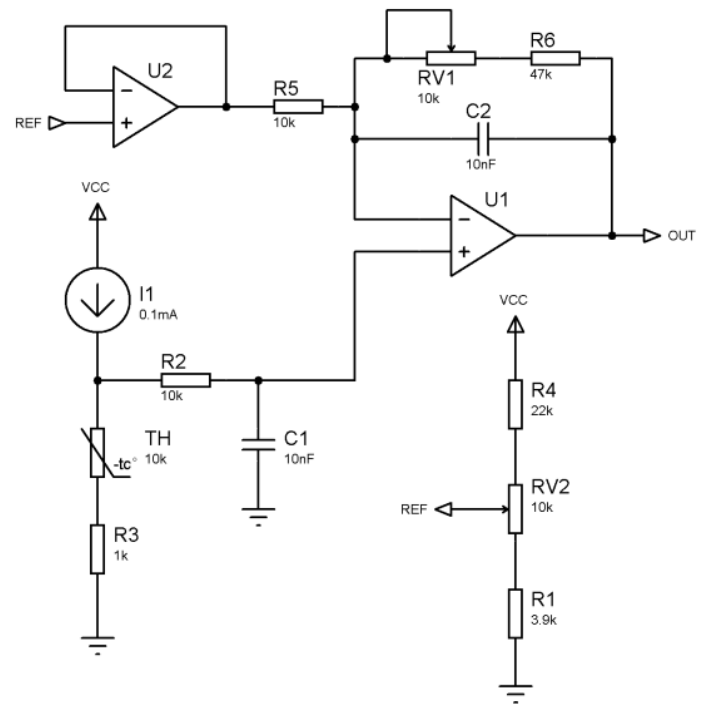

Fig 4: Temperature Sensor

The force sensor proposed on Fig. 2, one Force Sensing Resistor (FSR) with a round, 0.5 " diameter, sensing area from Interlink Electronics was selected. It varies its resistance depending on how much pressure is being applied to the sensing area. The greater the force, the lower the resistance. When no pressure is being applied to the FSR, its resistance will be larger than $1 \mathrm{M} \Omega$. This FSR can sense applied force 
anywhere in the range of $100 \mathrm{~g}-10 \mathrm{~kg}$. In Emopad this sensor is used for detecting weight shift and relative force and correlating them with sympathetic reactions, particularly those related with the flight-or-fight response, e.g., stress. The analog interface for the FSR implements a voltage divider. The value of fixed resistance should be chosen to give the output voltage the greatest "sweep" from $0-3.3 \mathrm{~V}$, assuming that the sensing range (resistance sweep) of the sensor is 0 $100 \mathrm{k} \Omega$. The Emopad circuitry is able to execute dynamic adjusts on the resistance value according to the voltage source adopted, using a digital potentiometer.

The heart rate and its variability are captured by PPG sensor SEN-11574 [31]. It combines a simple optical heart rate sensor with amplification and noise cancellation circuitry. Also, it sips power with $4 \mathrm{~mA}$ current. It can be applied to the earlobe or on the finger surface. Emopad embeds SEN-11574 to be used on the finger surface contact, without any obstructive artifact. Fig 5. shows SEN-11574 on Emopad prototype.

The A/D converter was set up to multichannel sampling, enabling MSP430's Low Power Mode 0 (LPM0) at $500 \mathrm{~Hz}$ sampling rate. This sampling rate was defined based on PPG and HVR requirements, according to [32].

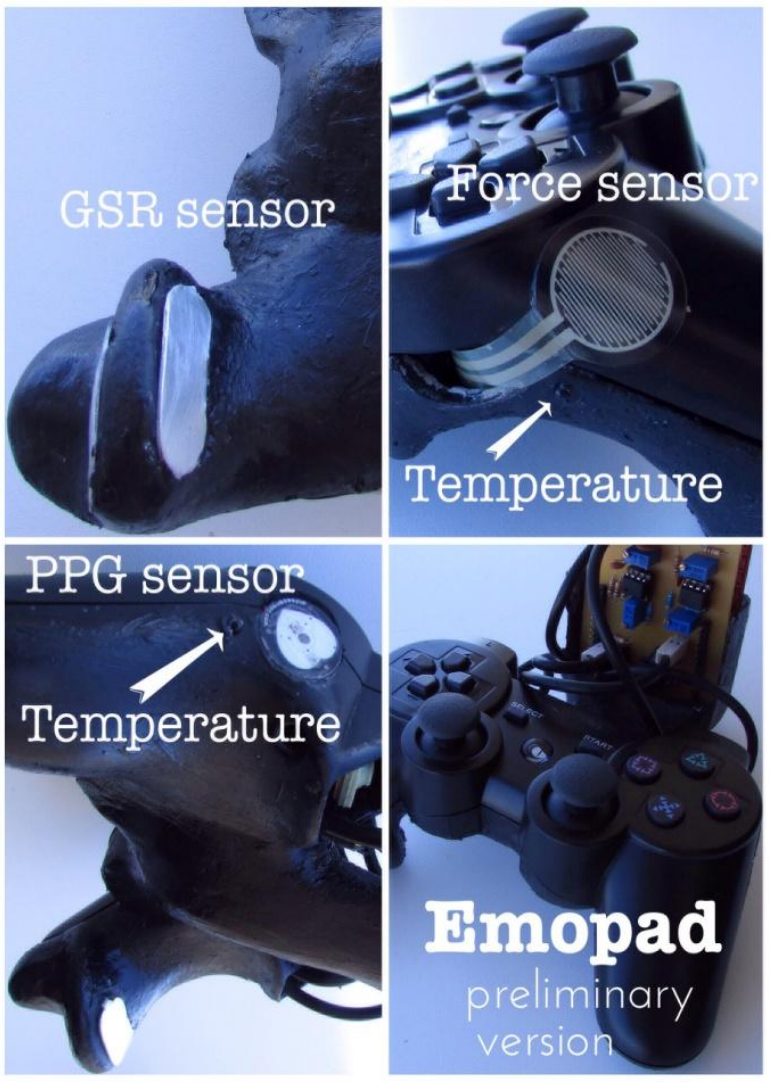

Fig 5: Prototype

\subsection{Dataset Storage and Usability Tests}

Fig. 6 shows structural description about dataset that was built to support the Emopad development. To support data exchange between Emopad Acquisition Systems (Fig.2) and PC software systems, a Matlab application was developed. It was called CollectPhys. CollectPhys enables Matlab's Data Acquisition Toolbox and its real-time plotter to receive, plot and save data from MSP430's USART module, embedded into Emopad hardware. Thus, wired and wireless serial communication can be configured to interconnect Emopad to videogame consoles. In the first Emopad version, a BlueSMiRF technology has been used to support wireless communication. The BlueSMiRF is the Bluetooth wireless serial cable replacement from SparkFun Electronics [33]. This modem works as a serial (RX/TX) pipe. Any serial stream from 2400 to $115200 \mathrm{bps}$ can be passed seamlessly from Emopad to videogame console. CollectPhys is also responsible to synchronize all sensors including those outside of Emopad, such as webcam, microphone, ambient temperature and humidity. On Fig. 7 is presented all sensor blocks that have been connected to data set.

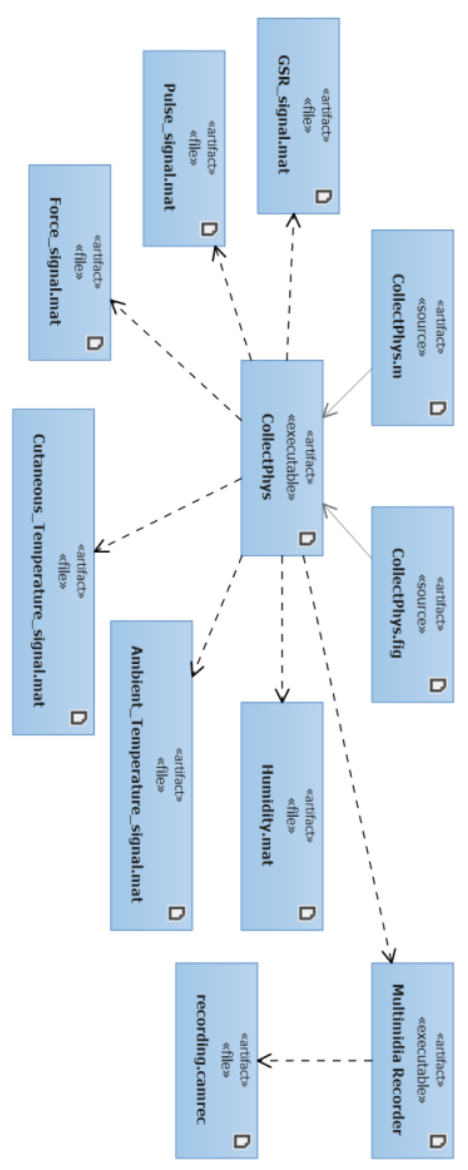

Fig 6: Dataset Artifacts Diagram

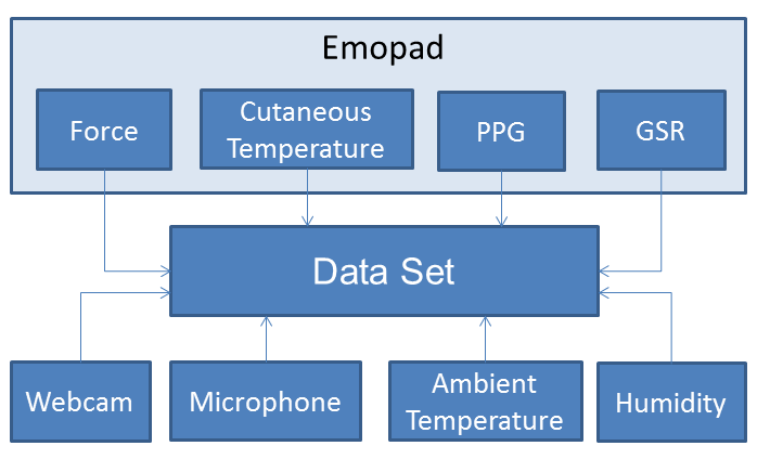

Fig 7: Usability Data Capture Systems (Block Diagram)

In order to apply usability tests certain elements should be chosen, including those related to the protocol requirements. The game choice was based on gameplay parameter. A linear 
gameplay allows reproducibility and, consequently, short time tests. A horror game was chosen because of its inherent and most common effect, the scare. Fright condition can be easily perceived in physiological markers and it can be verified and correlated with video recordings, e.g., those obtained from webcam. For each participant the maximum gameplay was 30 minutes and Death Unknown software [34] was used for usability tests. Death Unknown was supported by Intel Core i5 processor, AMD Radeon 7750 video board and 6GB RAM memory. At the same PC console Death Unknown and CollectPhys were run. Fig. 8 shows an instance of the usability tests.

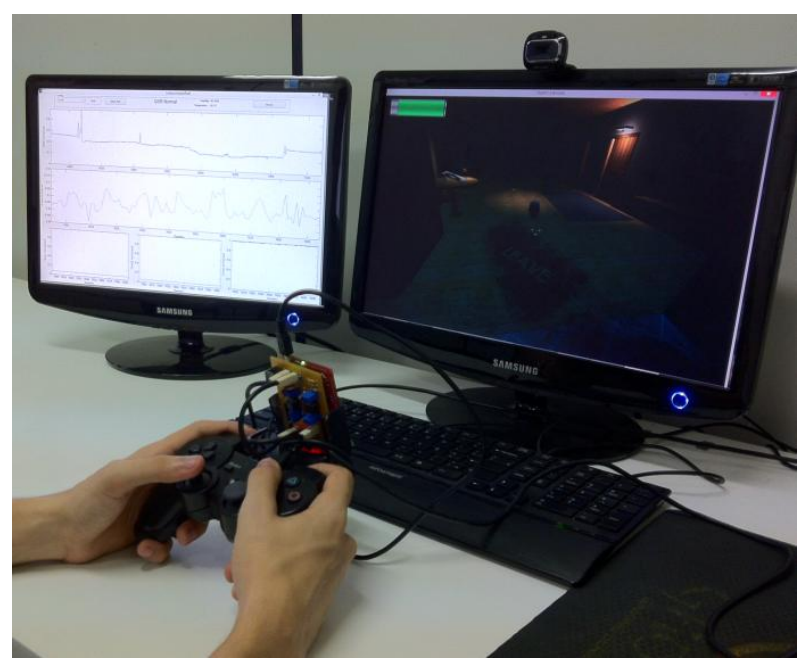

Fig 8: Usability Testing Environment

\subsection{The Analysis Tools}

Fig. 10 shows one screenshot obtained from analysis tools. They were conceived to evaluate and customize algorithms for real-time applications. Also, they are useful in visualizing sensors data on Matlab's environment and implements functionalities such as plotting, markers, labeling Emopad acquired data. These tools can be very convenient in clinical application of the Emopad.

\subsection{Emopad's Window-based Algorithm}

The event detection algorithm applied in acquired physiological signals is described in Fig. 9. The first part (Fig. 9) shows the filling and sliding of the window. In this example, each signal sample is stored into array called "Window". When it is completely full DETECTION method is invoked, which is responsible to detect alterations (abnormalities) on each window. DETECTION utilizes a modified version of the hill-climbing algorithm. Fig. 10 illustrates DETECTION applying it to GSR sample.

The variable "currentDifference" computes the difference between the maximum and minimum samples stored in each "window". Next, "differenceSum" and "countDifference" are incremented in order to establish the threshold level, which corresponds to arithmetic mean, moving on each window. It is important to filter many false positive detect peaks. Thus, if "currentDifference" is minor than "previousDifference" and "previousDifference" is major than threshold level, the search algorithm has to "climb", else it has to come down, meaning "climbing" should receive and return 0 .

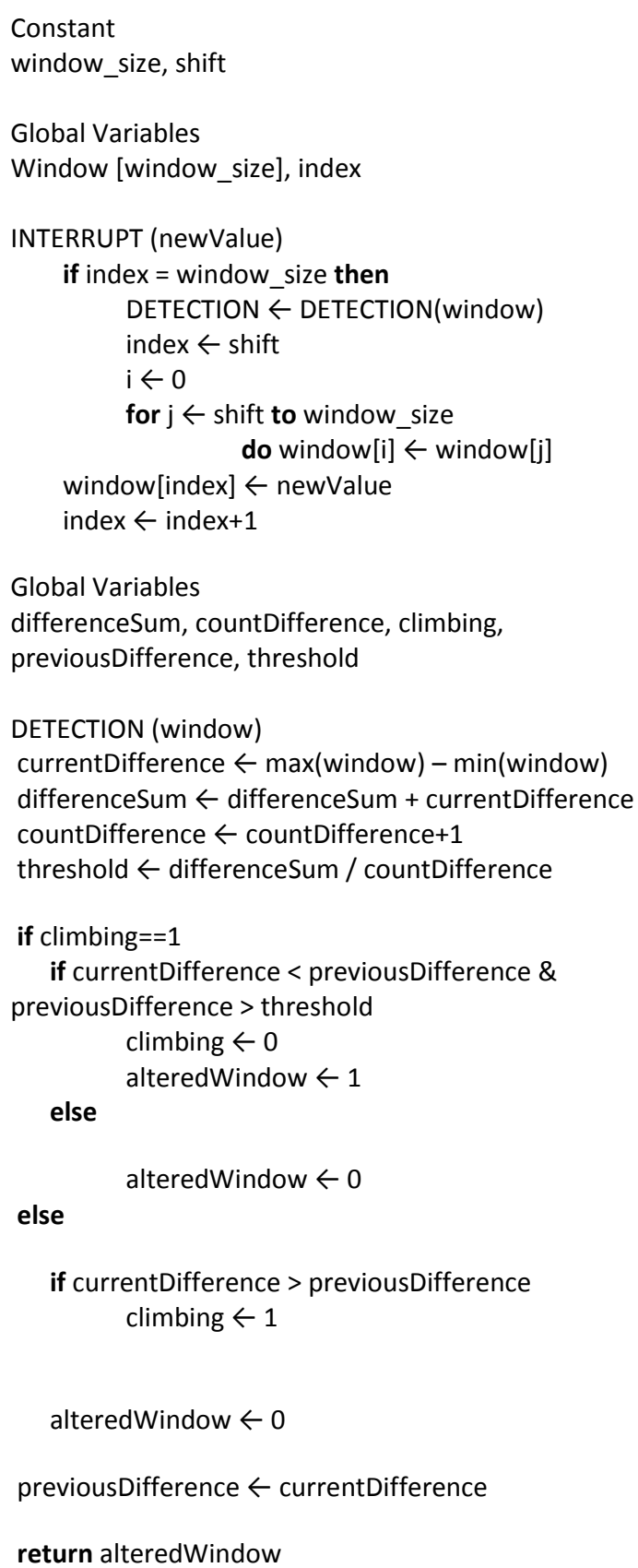

\section{Fig 9: The GSR Event Detection Algorithm}

In Fig. 10 the blue line plots the normalized GSR raw signal in the time domain (indexing sample number) while red line plots filtered GSR. The proposed algorithm reduces data dimensionality defining only one data sample to represent each window, highlighted in gray columns. This data sample is defined based on the difference between maximum and minimum encountered values. Also, it acts as rectifier preparing GSR signal to next stage. After that, hill-climbing algorithm can be applied in order to detect GSR peak points. 


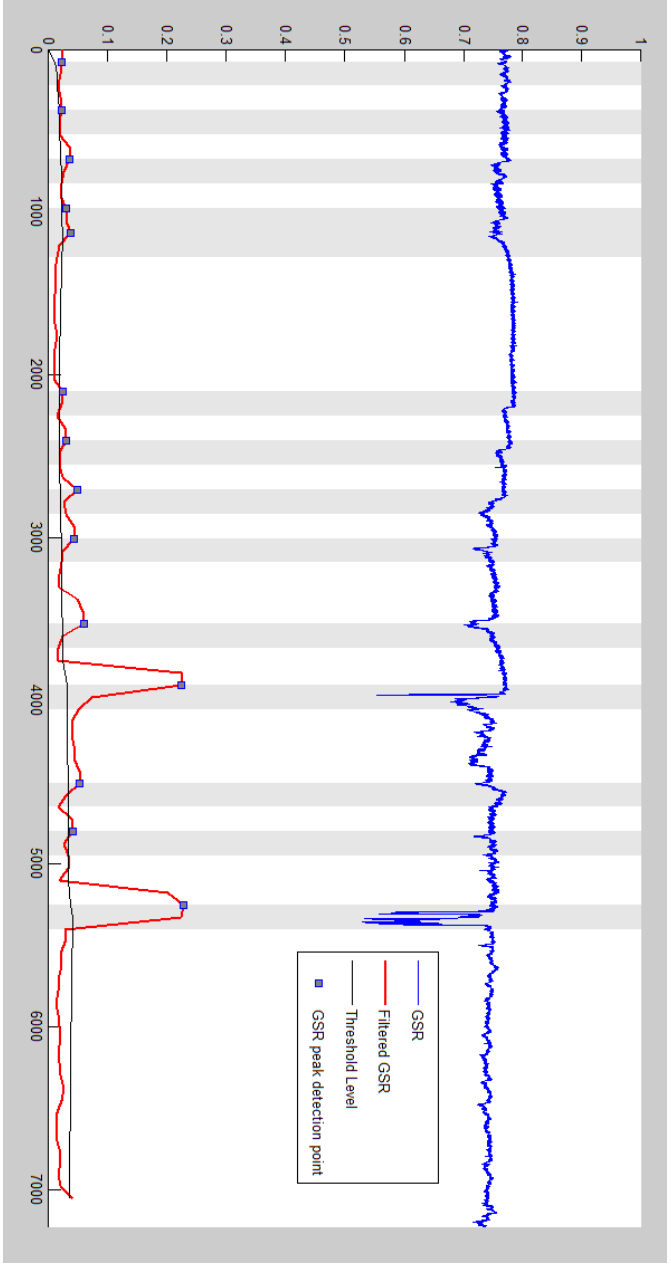

Fig 10: Analysis Tools Screenshot

\section{PRELIMINARY EVALUATION AND RESULTS}

The Emopad evaluation methodology was based on [35]. The usability tests were carried out in the 505E room in the Embedded Systems Laboratory, Department of Computing at Pontifical Catholic University of Goiás. The main aim of this activity was to collect and process physiological signals using the first version of Emopad prototype, presented in Fig. 5. Thus, the proposed window-based algorithm (Section 2.E) could be evaluated in real conditions. Also, ergonomic features have been assessed.

The participants were students from Computing Department, with a total of 31,15 female individuals of ages from 19 to 25 years old $(21.5 \pm 1)$ and 16 male individuals of ages from 19 to 25 years $(21.5 \pm 1.5)$. As described in Section 2.C, this paper proposes to induce fright in order to obtain a proper arousal in terms of physiological signals and simulate real conditions of using proposed technology. Female and male individuals differs have similar responses when the stress stimuli consist of specific actions in a very short period of time, e.g., an accident, an armed robbery, and the like [35]. Thus, we argue that the same horror game was a good choice for provoking scare in real conditions, although the 20 minutes (on average) for each gameplay.

Depending on the threshold level defined to window-based algorithm described in Section $2 \mathrm{E}$ is necessary to differ True Scare Detection (TSD) rate from True non-Scare Detection
(TNSD) rate. TSD corresponds to sensitivity statistical measurement while TNSD to the specificity. To compute TSD:

TSD $=$ True Positive/(True Positive + False Negative $)$

Where a True Positive means classifying as scared an individual GSR window which is indeed represents a real scare (seen on Webcam and correlated with HR peak), and False Negative means classifying as normal a GSR window which is corresponding to real scare. To compute FSD:

TNSD $=$ True Negatives/(True Negatives + False Positives)

Where a True Negative means classifying as non-scare an individual GSR window which is indeed represents a normal window (without GSR peaks) and False Positives means classifying as scared a GSR window which contains only normal samples, eg., relaxed. Fig. 11 shows TSD and TNSD for the twelve better window size/displacement size of the window, called "shifting size", showed in Table 1 while Table 2 presents the Confusion Matrix corresponding to the best configuration encountered in Table 1.

Table 1. Best TSD and TNSD rates obtained during the Emopad's usability testing for the proposed algorithm

\begin{tabular}{|c|c|c|c|}
\hline Window Size & Shifting Size & TSD(\%) & TNSD(\%) \\
\hline 140 & 30 & 100 & 92.4 \\
\hline 120 & 50 & 100 & 90.4 \\
\hline 120 & 20 & 100 & 93.9 \\
\hline $\mathbf{1 2 0}$ & $\mathbf{1 0}$ & $\mathbf{1 0 0}$ & $\mathbf{9 5 . 5}$ \\
\hline 110 & 40 & 100 & 90.5 \\
\hline $\mathbf{1 1 0}$ & $\mathbf{1 0}$ & $\mathbf{1 0 0}$ & $\mathbf{9 5 . 5}$ \\
\hline 90 & 10 & 100 & 94.3 \\
\hline 80 & 20 & 100 & 90.5 \\
\hline 80 & 10 & 100 & 93.6 \\
\hline 70 & 10 & 100 & 92.8 \\
\hline 60 & 10 & 100 & 92.3 \\
\hline 50 & 10 & 100 & 91.7 \\
\hline
\end{tabular}

Clearly, TSD and TNSD depend on threshold level, highlighted in Fig. 9. If threshold decreases the algorithm considers every output as an event related to scare. Thus, if threshold increases TSD increases while TNSD decreases. At this moment, the threshold level is computed based on arithmetic mean. Thus, a compromise must be reached by finding the best configuration of window size versus shifting size, when TSD are close to TSND. These indexes indicate where the errors in detecting false positives and false negatives are close. Fig. 11 shows TSD and TNSD performance related to the window size, when the shifting size is fixed, equals to 10 while Fig. 12 shows the same indexes when the displacement size of the window is changed to twenty samples. Both graphics are selected based on evidences encountered in Table 1. Blue line represents TSD percentage while red line describes TNSD performance. 


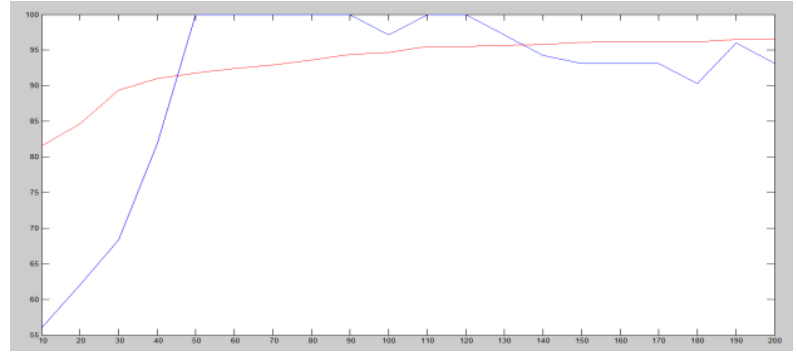

Fig 11: TSD and TNSD performance for shifting size equals to ten samples

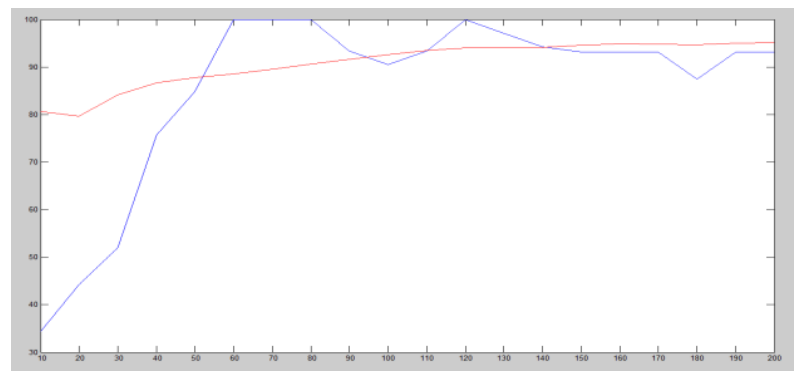

Fig 12: TSD and TNSD performance for shifting size equals to twenty samples

When the window size is very short errors become greater, even if the shifting size is changed to 20 samples (Fig. 12). The best performance is encountered when the window size is setup to 110 and the shifting size is setup to 10 samples (Fig.11), and window size equals to 120 , shifting size equals to 20, in Fig. 12. When the window size is bigger, more memory amount is required from embedded hardware. This is the assumption required for evaluating the performance of minors' window sizes. Fig. 11 and Fig. 12 reveal that the performance obtained from the window size when it is equals to 110 is almost the same (only $3 \%$ more) when compared to the windows size when it is equals to 50 . Table 2 shows the confusion matrix related to the best configuration obtained from Table 1 while Table 3 shows the confusion matrix when window size is halved. The "Scare" label counts the number of strong abnormalities for each processed window while "Non-Scare" label counts the number of processed windows without any strong abnormalities (high peaks). As it was verified in manual classification, almost always (95\%) there is a scare when a high peak occurs.

Table 2. Confusion matrix corresponding to the best configuration ( window size $=110 /$ shifting size $=10$ ) according to Table 1

\begin{tabular}{|c|c|c|c|}
\cline { 3 - 4 } \multicolumn{2}{c|}{} & \multicolumn{2}{c|}{ Actual } \\
\cline { 3 - 4 } \multicolumn{2}{c|}{} & Scare & Non-Scare \\
\hline \multirow{2}{*}{ Predicted } & Scare & 33 & 0 \\
\cline { 3 - 4 } & Non-Scare & 472 & 9605 \\
\hline
\end{tabular}

Table 3. Confusion matrix corresponding to the best configuration (window size $=50 /$ shifting size $=10$ ) according to Table 1

\begin{tabular}{|c|c|c|c|}
\cline { 3 - 4 } \multicolumn{2}{c|}{} & \multicolumn{2}{c|}{ Actual } \\
\cline { 3 - 4 } \multicolumn{2}{c|}{} & Scare & Non-Scare \\
\hline \multirow{2}{*}{ Predicted } & Scare & 32 & 0 \\
\cline { 2 - 4 } & Non-Scare & 828 & 9280 \\
\hline
\end{tabular}

\section{CONCLUSION AND FUTURE WORK}

On 24th April 2014, a Sony registered patent became public revealing a framework that should be developed for emotion recognition. It was called Multi-modal Sensor based Emotion Recognition And Emotional Interface [36]. "If the emotional state of the user or game player is known, a game or machine can dynamically adapt accordingly. For example, in a simple case, a game can become easier or harder for the user depending on the detected emotional state of the user". Sony's framework shares many hypotheses with Emopad project, although neither technology or algorithm solution was revealed or evidenced based on Sony's patent description. Thus, this paper proposes and presents a feasible platform, describing a complete solution.

The proposed scare-detection system that is embedded in traditional gamepad can be useful not only to entertainment applications, but its inherent characteristics, such as noninvasiveness and non-obstructiveness become it adequate to point-of-care monitoring.

Future works will concentrate in many aspects of Emopad, especially those related to data fusion algorithms in order to improve the pattern recognition accuracy based on multimodal sensor feature available on proposed platform. At this moment a classification algorithm is been developing to inform the game engine about arousal related to scare and other relevant emotional states. Also, a study involving many participants aims to reveal how temperature variations can be useful to recognize and classify emotional states.

\section{ACKNOWLEDGMENTS}

This study was supported by FAPEG (in portuguese, Fundação de Amparo à Pesquisa de Goiás) at Pontifical Catholic University of Goiás. Also, authors would like to thank all those involved in Emopad Project specially Jéssica Santillo, Erlan Lopes and Olegário Correa da Silva Neto for their valuable contributions.

\section{REFERENCES}

[1] R. Picard, "Affective computing", MIT Press. Boston, 1997.

[2] M. F. A. Carvalhaes, A. F da Rocha, M. F. Vieira and T. M. G. de A. Barbosa, "Affective embedded systems: a requirement engineering aproach", International Journal of Computer Trends and Technology (IJCTT), v. 8, pp. 70-75, Feburary 2014.

[3] D. Bersak, G. McDarby, N. Augenblick, et al., "Intelligent biofeedback using an immersive competitive environment," in Proceedings of the UbiComp Workshop on Designing Ubiquitous Computing Games, 2001.

[4] V. H. M. Gomide, P. A. Valle, J. O. Ferreira, J. R. G. Barbosa, A. F. da Rocha and T. M. G. de A. Barbosa, "Affective crowdsourcing applied to usability software", International Journal of Computer Science and Information Technologies (IJCSIT), Vol. 5 (1) , 2014, 575-579. doi: 10.14445/22312803/IJCTT-V8P113.

[5] J. Schumm, C. Setz, M. Bächlin, M. Bächler, B. Arnrich, G. Tröster, "Unobtrusive physiological monitoring in an airplane seat", Personal and Ubiquitous Computing, V. 14 (6), 2010, pp. 541-550. doi: 10.1007/s00779-0090272-1.

[6] M. Z. Poh, k. Kim, K., A. D. Goessling,N. C. Swenson, R. W. Picard, "Heartphones: sensor earphones and mobile application for non-obtrusive health monitoring," Proceedings of the 13th IEEE International Symposium 
on Wearable Computers, Linz, Austria, 2009, pp. 153154

[7] M. Z. Poh, D. J. McDuff and R. Picard, "Advancements in Non-contact, Multiparameter Physiological Measurements Using a Webcam," IEEE Transactions on Biomedical Engineering, vol.58, no.1, pp. 7-11, Jan 2011. doi: 10.1109/TBME.2010.2086456.

[8] R. Fletcher, K. Dobson, M. S. Goodwin, H. Eydgahi, D. Wilder-Smith, D. Fernholz, Y. Kuboyama, E. Hedman, M. Z. Poh, R. W. Picard, "iCalm: wearable sensor and network architecture for wirelessly communicating and logging autonomic activity," IEEE Transactions on Information Technology in Biomedicine, vol.14, no.2, pp.215-223, March $2010 . \quad$ doi: 10.1109/TITB.2009.2038692.

[9] F. Arafsha, K. M. Alam and A. El Saddik, "EmoJacket: consumer centric wearable affective jacket to enhance emotional immersion", Proceedings of International Conference on Innovations in Information Technology (IIT), 2012, pp. 350-355.

[10] R. Fernandez and R.W. Picard (2003), "Modeling Driver's Speech under Stress," Speech Communication, Volume 40, pp. 145-159.

[11] J. Hernandez, D. McDuff, X. Benavides, J. Amores, P. Maes and R. Picard, "AutoEmotive: Bringing Empathy to the Driving Experience to Reduce Stress," To appear as a provocative work in Designing Interactive Systems (DIS), Vancouver, Canada 2014.

[12] K. Williams, J. Peters and C. Breazeal, "Towards Leveraging the Driver's Mobile Device for an Intelligent, Sociable In-Car Robotic Assistant," IEEE Intelligent Vehicles Symposium, 23-26 Jun. 2013.

[13] S. O. Adalgeirsson and C. Breazeal, "MeBot: A robotic platform for socially embodied telepresence" Proceedings of 5th ACM/IEEE International Conference on Human-Robot Interaction (HRI), 2010.

[14] M. Z. Poh, D. J. McDuff and Picard, R.W., "A Medical Mirror for Non-Contact Health Monitoring," ACM SIGGRAPH Emerging Technologies, Aug 2011.

[15] J. Hernandez, I.Riobo, A. Rozga, G. D. Abowd and R. W. Picard, "How Easy Are Children to Engage during Child-Adult Play? Using Electrodermal Activity as Identifier", To appear in the Extended Abstract of IMFAR, Alanta, USA, 2014.

[16] T. Teixeira, T. Kaliouby and R. Picard, "Why, When and How Much to Entertain Consumers in Advertisements? A Web-based Facial Tracking Field Study," Marketing Science (To Appear) 2014.

[17] M. Eckhardt, C. Ferguson and R. W. Picard, "A Platform for Creating Stories Across Digital and Physical Boundaries," Proceedings of the 8th International Conference on Tangible, Embedded and Embodied Interaction. ACM, 2014

[18] L. E. Nack, M. Kalyn, C. Lough and R. L. Mandryk Biofeedback Game Design: Using Direct and Indirect Physiological Control to Enhance Game Interaction" Proceedings of ACM Conference on Human Factors in Computing Systems (CHI), 2011.

[19] K. Schaaff and M. T. P. Adam, "Measuring Emotional Arousal for Online Applications: Evaluation of Ultra-
Short Term Heart Rate Variability Measures" Proceedings of Humaine Association Conference on Affective Computing and Intelligent Interaction, 2013.

[20] A. Parnandi, Y. Son amd R. Gutierrez-Osuna, "A Control-Theoretic Aproach to Adaptive Physiological Games" Proceedings of Humaine Association Conference on Affective Computing and Intelligent Interaction, 2013.

[21] M. W. Kennedy, J. P. Shimiedeler, C. R. Crowell, M. Villano, A. D. Striegel and J. Kuitse, "Enhanced Feedback in Balance Rehabilitation using the Nintendo Wii Balance Board" Proceedings of 13th IEEE International Conference on e-Health Networking, Applications and Services, 2011.

[22] L. E. Nack, M. Kalyn, C. Lough and R. L. Mandryk Biofeedback Game Design: Using Direct and Indirect Physiological Control to Enhance Game Interaction" Proceedings of ACM Conference on Human Factors in Computing Systems (CHI), 2011.

[23] Neurosky - Body and Mind: Throw Trucks. Online: http://store.neurosky.com/products/throw-trucks-withyour-mind?mc_cid=6c3dfb6fe0\&mc_eid=53dde7647e/, April, 2014.

[24] J. Hsu, "Game Controller Senses Players' Excitement Levels", Online: http://spectrum.ieee.org/techtalk/consumer-electronics/gaming/game-controllersenses-players-excitement-levels, April, 2014.

[25] Shapeways. Online: http://www.shapeways.com.

[26] STERN, R.M., RAY, W.J.\& QUIGLEY, K.S., Psychophysiological Recording. (2nd ed.), Oxford University Press, New York, 2001.

[27] Lang, P. J.The Emotion Probe. American Psychologist, 50(5), pp.372-385, 1995.

[28] R. W. Picard, S. Fedor \& Y. Ayzenberg, "Multiple Arousal Theory and Daily-Life Electrodermal Activity Asymmetry" Emotion Review, (To Appear) 2014.

[29] J. Bishop, "Thermistor temperature transducer-to-ADC application" in Analog Applications Journal, Texas Instruments Incorporated.

[30] Epcos, "NTC thermistors for temperature measurement", Mar 2013.

[31] PulseSensor. Online: http://pulsesensor.myshopify.com/, April, 2014.

[32] J. G. Webster, "Medical Instrumentation Application and Design", 4th ed., Wiley, 2009.

[33] Sparkfun, "Bluetooth Modem - BlueSMiRF”.Online: https://www.sparkfun.com/products/12582.

[34] Game Jolt, "Death Unknown". Online: http://gamejolt.com/games/other/death-unknown-v-12/16329/".

[35] A. S. Sierra, C. S. Ávila, J. G. Casanova and G. B. del Pozo, "A Stress-Detection Based on Physiological Signals and Fuzzy Logic", IEEE Transactions on Industrial Electronics, v. 58, n. 10, october 2011.

[36] O. Kalinli-Akbacak, "Multi-modal sensor based emotion recognition and emotional interface". Online: http://www.google.com/patents/US201401125 